\title{
A diversity-sensitive long-term care for gay and lesbian elders in need of care - Results of the research project GLESA
} Eine diversitätssensible Pflege für schwule und lesbische
Pflegebedürftige - Ergebnisse des Forschungsprojekts GLESA

\author{
Ralf Lottmann ${ }^{1 *}$, Ingrid Kollak' \\ ${ }^{1}$ Alice Salomon Hochschule Berlin, 12627 Berlin, Germany \\ *lottmann@ash-berlin.eu
}

Received 21 April 2017, accepted 17 February 2018

\begin{abstract}
This paper presents results of the research project "Gleichgeschlechtliche Lebensweisen und Selbstbestimmung im Alter" (GLESA) by the Alice Salomon University of Applied Sciences, Berlin, and the Berlin School of Economics and Law concerning the needs of gay and lesbian elders regarding long-term care. The main focus is on the expectations and perceptions of long-term care (facilities) of the interviewees living in a gay housing project in Berlin, Germany. The study is based on 26 interviews: with eleven gay, one lesbian and three heterosexual tenants - two of them lived in a shared community with long-term care services. Another eleven interviews were conducted with experts (five cis-female, four cis-male and two transgender) working in social and health services (social workers, carers and psychologists). The data was gathered via problem-centered interviews (Witzel 2000) and analysed with Mayrings' (2007) qualitative content analysis. Long-term care aspects were one out of five dimensions of the analysis. The study illustrates the discomfort of LGB elders regarding regular care services. The interviewees prefer $L G B\left(T^{*}\right)$-friendly facilities, in part because they demonstrate overt signs of diversity and promise a high competence of $L G B\left(T^{*} \mid\right)$ personnel in terms of self-determination, awareness, visibility and knowledge about LGBT*I communities. Finally, the authors advocate enhancing the concept of culture-sensitive longterm care according to diversity-sensitive aspects. A better understanding of diversity will help to better consider individuality and biographies in long-term care (facilities) and to support the social inclusion of LGB(T*I) elders in need of care.
\end{abstract}

\begin{abstract}
Dieser Artikel stellt ausgewählte Ergebnisse des Forschungsprojekts „Gleichgeschlechtliche Lebensweisen und Selbstbestimmung im Alter" (GLESA) derAlice Salomon Hochschule Berlin und der Hochschule für Wirtschaft und Recht Berlin im Hinblick auf Bedürfnisse von schwulen und lesbischen Senior*innen im Bereich der Pflege vor. Dabei werden Erwartungen und Besonderheiten bei der Pflege von lesbischen und schwulen Senior*innnen und das Erleben von Pflegesituationen aus der Perspektive von Bewohner*innen eines vorwiegend schwulen Wohnprojekts in Berlin-Charlottenburg präsentiert und diskutiert. Die Studie basiert auf 26 Interviews. Diese wurden mit elf schwulen, einer lesbischen und drei heterosexuellen Bewohner*innen geführt - zwei interviewte Personen wohnten zum Zeitpunkt der Befragung in einer „Pflege-WG“ innerhalb des Wohnprojekts. Weitere elf Interviews wurden mit Expert*innen (fünf cis-weiblichen, vier cis-männlichen und zwei transidenten Mitarbeitenden) in der Gesundheits- und Sozialversorgung (Sozialarbeiter*innen, Pflegepersonen, Psycholog*innen) geführt. Erhoben wurden die Daten mit problemzentrierten Interviews nach Witzel (2000). Die Datenanalyse erfolgte mit der qualitativen Inhaltsanalyse nach Mayring (2007). Pflegerische Aspekte waren eine von fünf Sachdimensionen der Analyse. Diese illustriert die Vorbehalte von schwulen und lesbischen Senior*innen gegenüber Regeldiensten der Altenhilfe. Sie ziehen LSB(T*I)-freundliche Einrichtungen klassischen Pflegesettings vor, u.a. weil sie nach außen sichtbare Signale der Diversität setzen und LSB(T*I)-Personal eine hohe Kompetenz bei der Gewährleistung von Selbstbestimmung, Sichtbarkeit und Lebensweltkenntnissen zuschreiben. Darüber hinaus plädieren die Autor*innen dafür, das Konzept der kultursensiblen Altenpflege im Hinblick auf diversitätssensible Gesichtspunkte weiter zu entwickeln. Ein Verständnis für Diversität erlaubt es den unterschiedlichen Berufsgruppen, die Individualität und Biografie pflegebedürftiger LSB( $\left(T^{*}\right)$-Senior*innen besser zu berücksichtigen und deren soziale Teilhabe zu unterstützen.
\end{abstract}

\section{Keywords}

Diversity - LGBT*I - Long-term Care - Gay - Lesbian - Homosexuality

\section{Keywords}

Diversität - LSBT*I - Pflege - schwul - lesbisch - Homosexualität

\section{EINLEITUNG}

Gibt es Besonderheiten bei der Pflege von älteren, pflegebedürftigen Lesben und Schwulen?' Das Forschungsprojekt „Gleichgeschlechtliche Lebensweisen und Selbstbestimmung im Alter" (GLESA) ist als eines der ersten in Deutschland dieser Frage nachgegangen und hat damit neue Einblicke in das Schnittfeld Alternsforschung, Pflegewissenschaften und Queer Studies geschaffen. Den Anlass für die Studie gab das 2012 eröffnete Wohn- und 
Pflegeprojekt „Lebensort Vielfalt“ in Berlin $(\mathrm{LoV}) .{ }^{2} \mathrm{LoV}$ ist ein Mehrgenerationenhaus mit 24 Privatwohnungen und einer betreuten „Pflege-WG“ für schwule Männer mit Pflegebedarf und Demenz. In der ersten Etage hat die Trägerorganisation, die Schwulenberatung Berlin, (SB) ihre Räume. Im Erdgeschoss befinden sich ein Restaurant, eine Bibliothek, eine Concierge und Gruppenräume (LoV, 2017).

Im Rahmen unserer GLESA-Studie interessierten Fragestellungen zum Wohnprojekt wie: Charakteristika des LoV, Kommunikation und Teilhabe im LoV sowie Altersbilder der Bewohner*innen. ${ }^{3}$ In dieser qualitativen Studie wurden zudem auch Aspekte für eine ,gute Pflege" von Lesben, Schwulen, Bisexuellen sowie trans* und intergeschlechtlichen Senior*innen (LSBT*I) im LoV untersucht. Die Auswertung der Ergebnisse steht unter der Fragestellung: In welcher Weise können aus der Sicht schwuler und lesbischer Bewohner*innen des LoV Besonderheiten für eine gute Pflege plausibel gemacht werden?

Als rechtlicher und theoretischer Hintergrund sowie zur Unterstützung der Datenanalyse beziehen wir uns auf die „Charta der Rechte hilfe- und pflegebedürftiger Menschen" (Pflege-Charta) sowie auf das Konzept der kultursensiblen Altenpflege. Die Pflege-Charta spricht an einer Stelle explizit die sexuelle Orientierung an:

Grundsätzlich hat jeder Mensch - unabhängig vom Alter und unabhängig vom Ausmaß des Pflegeund Hilfebedarfs - das Recht auf Sexualität, auf Respektierung seiner geschlechtlichen Identität und seiner Lebensweise. Niemand darf Sie aufgrund Ihrer geschlechtlichen Orientierung diskriminieren. (Artikel 3 „Privatheit“, Abschnitt: „Respektierung von Sexualität, geschlechtlicher Orientierung und Lebensweise.“)

Im „Leitfaden zur Selbstbewertung auf Grundlage der Pflege-Charta der Rechte hilfe- und pflegebedürftiger Menschen - Eine Handlungshilfe zur Umsetzung der Pflege-Charta in stationären Pflegeeinrichtungen" des Bundesministeriums für Familie, Senioren, Frauen und Jugend (Konkret Consult Ruhr, 2008) werden dazu weitere Fragen für die Selbstbewertung von Einrichtungen formuliert, von denen eine die sexuelle Identität betrifft:

Was wird in der Einrichtung getan,

... um Bewohner/innen und Mitarbeiter/innen vor Diskriminierung z.B. aufgrund ihrer/seiner Herkunft oder sexuellen Identität zu schützen? (Konkret Consult Ruhr, 2008, p. 10).

Das Konzept der kultursensiblen Altenpflege bezieht sich auf die Pflege-Charta, die vor allem Migrant*innen fokussiert (Dömling, 2012). Bei der Auswertung unserer Ergebnisse gehen wir daher auch der Frage nach, inwiefern das Konzept der kultursensiblen Pflege auch auf lesbische und schwule Pflegebedürftige übertragbar ist.

\section{Forschungsstand}

Die Situation von älteren und pflegebedürftigen LSBT*I erlangt international zunehmend Bedeutung - sowohl in der LSBT*I-Community als auch in der Alterns- und Pflegeforschung. Doch gibt es nur wenige empirische Studien zur Situation von LSBT*I-Personen im Alter (Lautmann, 2016; Lottmann \& Lautmann, 2015; Reimann \& Lasch, 2006). Insbesondere die Bedürfnisse von hochaltrigen und pflegebedürftigen LSBT*IPersonen gelten weitgehend als Neuland der Alterns- und Pflegeforschung - obwohl ein verbessertes Verstehen der sozialen, gesundheitlichen und pflegerischen Bedürfnisse bei den möglichen Besonderheiten von LSBT*-Älteren unerlässlich ist, um adäquate kommunale Versorgungsstrukturen zu entwickeln (Adelman, 2010). Internationale Studien stellen einige allgemeine Risiken fest: LSBT*I-Ältere sind mit einer höheren Wahrscheinlichkeit kinderlos und alleinlebend (White \& Gendron, 2016; Adelman, 2010). Diese Merkmale gelten aufgrund der Unterstützungspotenziale als relevant für eine Gewährleistung einer guten Pflegeund Lebensqualität von LSBT*I-Senior*innen sowie als Beitrag zur Verhinderung von Isolation im Alter (van de Meerendonk, van de Adriansen, Vanwesenbeeck, 2003; Brennan-Ing, Liz, Larson, Karpiak, 2014; Adelman, 2010). Internationale, aber auch einige deutsche Studien berichten ferner von einem beständigen Unbehagen dieser Bevölkerungsgruppen gegenüber regulären Angeboten der Pflegedienste aufgrund befürchteter homophober Reaktionen (Schröder, Schondelmayer, Scheffler, 2012; Gardner, de Vries, Mockus, 2013). Über die Situation von LSBT*I-Senior*innen ist bei sozialen Diensten und der Altenhilfe nur wenig bekannt (FredriksenGoldsen \& Muraco, 2010; Schröder \& Scheffler, 2015; Reimann \& Lasch, 2006). In einer Münchner Befragung (Unterforsthuber \& Franz, 2004) verneinten über 90 Prozent der schwulen, lesbischen und bisexuellen Befragten, dass die vorhandenen Einrichtungen im Umgang mit Homosexuellen kompetent seien. Es gibt zahlreiche Berichte von Betroffenen über erlebtes Unwohlsein und Unverständnis in herkömmlichen Pflege- und Betreuungseinrichtungen (vgl. Gerlach, 2002; Jackson, Johnson, Roberts, 2008). Sogar eine Gefahr der Verwahrlosung wird bei schwulen und lesbischen Pflegebedürftigen gesehen, wenn sie sich aufgrund von inadäquaten Versorgungs- und Beratungssituationen zurückziehen (ebd.). Laut Bochow (2008, 2005, 
p.348ff.)befürchtenHomosexuelle(hier:schwuleMänner) im Alter bzw. in stationären Pflegeeinrichtungen, die sexuelle Orientierung und den Lebensstil verheimlichen zu müssen. Diese Untersuchungen widersprechen den Inhalten der Pflege-Charta in erheblichem Maße und machen eine entsprechende Untersuchung, wie unsere GLESA-Studie, sinnvoll.

Erstmals wurde mit einer Broschüre von Rubicon e. V. (gefördert durch das Ministerium für Gesundheit, Emanzipation, Pflege und Alter des Landes NordrheinWestfalen) im Jahr 2014 das Konzept der kultursensiblen Altenpflege auf lesbische und schwule Menschen konzeptionell übertragen und für die Pflegepraxis übersetzt (Stummer, 2014). Als zentrale Kennzeichen der Kultursensibilität wurden bei dem Projekt (a) das Interesse an der (anderen) Kultur, (b) das Wissen über die andere Kultur und (c) die Haltung benannt, die kulturelle Besonderheiten anerkennen und erkennen kann (ebd., p. 6). Paillon (2010) identifiziert darüber hinaus die Bedeutung der sozialen Netzwerke, die Berücksichtigung des „Heimatgefühls“, die Sprache, die Wertschätzung und die Angehörigenarbeit als Schwerpunkte einer kultursensiblen Altenpflege, denen in der Pflegepraxis seitens der Pflegepersonen im besonderen Maße Beachtung beigemessen werden sollte. „Kultursensibilität ist in Bezug auf pflegebedürftige Homosexuelle noch nicht geläufig" summiert Stummer (2014).

Wir möchten an dieser Stelle grundsätzlich fragen, ob es sich bei den LSBT*I Lebenswelten überhaupt nur um „eine“ und um ,eine andere Kultur“ handelt. Bereits in der Debatte über die kultursensible Pflege zeigte sich, wie schillernd der Begriff der Kultur ist und dass unterschiedliche Ebenen der Identität und Vielfalt unbeachtet bleiben, wenn z.B. die Rede von der Pflege „türkischer oder muslimischer Patienten“ ist. Sexuelle Orientierung, aberauch Alter, Geschlecht,Einkommenund soziale Herkunft, um einige Beispiele zu geben, werden bei einer solchen „kulturellen Verortung“ nicht beachtet. Anders herum können soziale Unterscheidungsmerkmale, wie z.B. Herkunft, Ausbildung, Beruf und Familienstand nicht unter dem Begriff der Kultur subsumiert werden. Ebenso ist es wenig hilfreich, von z.B. „einer“ lesbischen Kultur auszugehen. Auch in der Gruppe von Minderheiten gibt es soziale, ökonomische, lebensweltliche Vielfalt. Nicht zuletzt würde es bei der zu fördernden kulturellen Sensibilität, wie sie oben vorgeschlagen wird, darauf hinauslaufen, die „eine Kultur“ als gegeben und bekannt vorauszusetzen und für ein Erkennen und Verstehen „einer anderen Kultur“ zu werben. Was eine Person als „anders“ oder „fremd“" wahrnimmt, sagt viel über die Person selbst aus. Kollak \& Küpper fragten darum in ihrem Aufsatz zur „Multikulturalität in der Pflege“ bereits 1997 danach, wer oder was einer Pflegefachperson denn fremd erscheint: ,ein türkischer Vegetarier, eine niederländische
Nonne oder ein senegalesischer Homosexueller" (p. 120). Sie verwiesen mit dieser Frage darauf, „dass Pflege einerseits von einem historischen, sozialen und kulturellen Verständnis geprägt und andererseits auf der mikrosoziologischen Ebene immer mit der Komplexität von Beziehungen konfrontiert wird" (p. 120).

Inwieweit sich also von „den“ lesbischen und schwulen Pflegebedürftigen sprechen lässt und in welcher Weise sich Erkenntnisse über eine kultursensible Pflege auf die Gruppe der Befragten übertragen lassen, erörtern wir in der Diskussion des Datenmaterials der GLESAUntersuchung. Wir werden ebenso diskutieren, inwiefern die Charakteristika des LoV für eine mögliche Pluralisierung der pflegerischen Versorgung hilfreich sind.

\section{METHODISCHES VORGEHEN}

Dem noch niedrigen Forschungsstand entsprechend, wählte die Studie ein qualitatives und exploratives Design. Explorativ, da es zur Bedürfnislage älterer, pflegebedürftiger LSBTI-Leute bislang kaum spezifische Annahmen gibt und für die Untersuchung der konkreten Forschungsfragen keine getesteten Instrumente zur Verfügung stehen. Gleichzeitig nutzt die Studie die Stärken qualitativer Forschung, um die vielfältigen Perspektiven von LSBT*I sichtbar zu machen.

Als Erhebungsinstrument wurde das problemzentrierte Interview nach Witzel (2000) gewählt. Um ein möglichst breites und differenziertes Bild zum LoV zu erhalten, wurden sowohl Bewohner*innen als auch Expert*innen befragt. 17 von 32 Bewohner*innen konnten interviewt werden. Die Interviewleitfäden für Bewohner*innen und Expert*innen waren in folgende Themenblöcke (Witzel, 2000) unterteilt: Altersbilder, Besonderheiten des LoV, Rolle des Personals und Bedarfe von LSBT*I Bewohner*innen zu Wohnen und Pflege. Die Fragen waren offen formuliert und orientierten sich an den in der Pflegecharta angesprochenen Patientenrechten.

Der Zugang erfolgte über die Teilnahme der Forscher*innen an Mieter*innentreffen im LoV und gezielten Anschreiben an Bewohner*innen, die nicht an den Treffen teilgenommen hatten. Die Interviews mit den Bewohner*innen wurden in den Privatwohnungen der Bewohner*innen oder in Gemeinschaftsräumen des LoV geführt, die Interviews mit den Expert*innen in Büroräumen der Schwulenberatung (SB). Alle Interviews wurden von einer cis-weiblichen, lesbischen Mitarbeiterin und einem cis-männlichen, schwulen Mitarbeiter des GLESA-Forschungsprojekts durchgeführt und mit einem Tonbandgerät aufgezeichnet. ${ }^{4}$

Ein Votum der Ethikkommission musste nicht eingeholt werden, weil keine Interventionen vorlagen und die Teilnehmenden selbstbestimmt über Zustimmung oder 
Ablehnung der Interviewanfrage entscheiden konnten. Einwilligungserklärungen aller Befragten wurden eingeholt; auf die allein wissenschaftliche Nutzung der Daten wurde verwiesen.

Für das Sample konnten unter den Bewohner*innen 17 Personen gewonnen werden, von denen zum Zeitpunkt der Befragung $15 \mathrm{im} \mathrm{LoV}$ und zwei in der „Pflege-WG“ wohnten.

Von den 17 geführten Bewohner*inneninterviews wurden 15 in die Analyse aufgenommen und nach Transkriptionsregeln von Hoffmann-Riem (1984, p. 331) transkribiert. Bei der Auswahl der zu analysierenden Interviews mit Bewohner*innen wurde versucht, die Diversität der Bewohner*innenschaft abzubilden. So war uns wichtig, die im LoV unterrepräsentierte Gruppen der Frauen und der Bewohner*innen mit geringem Einkommen zu berücksichtigen.

Von den befragten Bewohner*innen sind zum Zeitpunkt der Interviews elf schwule Männer im Alter von 33 bis 76 Jahren und drei heterosexuelle Frauen sowie eine lesbische Frau im Alter von 65 bis 72 Jahren. Einige der befragten Bewohner*innen sind gesundheitlich (zum Teil stark) eingeschränkt, jedoch nicht pflegebedürftig. Die beiden Befragten aus der „Pflege-WG“ des LoV sind pflegebedürftig im Sinne des SGB XI und 65 bzw. 73 Jahre alt.

Als Expert*innen wurden Mitarbeitende in der Gesundheits- und Sozialversorgung (Berufsgruppen: Sozialarbeiter*innen, Pflegepersonen, Psycholog*innen etc.) definiert, die langjährig Funktionen in der Schwulenberatung (SB) und in dem mit dem LoV kooperierenden ambulanten Pflegedienst ausfüllen (Meuser \& Nagel, 2005, p. 80f.). Alle für den LoV verantwortliche/n Mitarbeiter*innen der SB sowie Expert*innen zweier Kooperationspartner (Pflegedienst und Antidiskriminierungsstelle des Landes) der SB wurden interviewt. Von diesen sind zehn Interviews transkribiert worden und in die Analyse der Daten eingegangen. Die Gruppe der befragten Expert*innen bestand aus fünf cis-weiblichen (eine lesbisch, vier heterosexuell), vier cis-männlichen (schwul) und einem transidenten Hauptamtlichen. Die Alterspanne bei den Expert*innen (Hauptamtliche) betrug zum Zeitpunkt der Befragung 43 bis 58 Jahre. $^{5}$

Für die Auswertung der Interviews wurde maßgeblich die qualitative Inhaltsanalyse nach Mayring (2007) herangezogen. Bei der Auswertung der Interviews wurde zunächst versucht, das Spezifische der jeweiligen Interviews darzustellen und Unterschiede kontrastiv hervorzuheben, um die Bandbreite der Aussagen möglichst adäquat abbilden zu können. Hierfür wurde ein Codierleitfaden angefertigt, der nach den o.g. Themenblöcken (bzw. Sachdimensionen) strukturiert wurde. Aufgrund der identischen Erhebungsinstrumente für Bewohner*innen und Expert*innen wurden die jeweiligen Sachdimensionen und Kategorien in einen Codierleitfaden integriert. Die Aussagen im nachfolgenden Ergebnisteil werden jedoch weiterhin nach Expert*innen und Bewohner*innen unterschieden. Wenn die Ergebnisse sich hinsichtlich der Befragtengruppe unterscheiden, wurde dies kenntlich gemacht.

\section{ERGEBNISSE}

Im Rahmen der GLESA-Untersuchung konnten einige Erfordernisse und Bedarfe für eine gute Pflege herausgearbeitet werden, die spezifischen Kriterien einer Pflege von pflegebedürftigen schwulen und lesbischen Personen kennzeichnen. Sie ergänzen die grundlegenden Kriterien einer zufriedenstellenden Versorgung. Diese Kriterien einer guten Pflege richten sich auf die professionellen Haltungen und fachlichen Fähigkeiten von Pflegepersonen gegenüber Pflegebedürftigen. Diese äußern sich bspw. im Konzept einer empathischen Pflege (Bischoff-Wanner, 2002) sowie im personenzentrierten Ansatz (Kitwood, 2000) und in der Validation (Feil, 1967, 2014) gegenüber bestimmten Patient*innengruppen oder in der Anwendung bestimmter Verfahren, wie z.B. dem Care- und Case-Management in der Pflege (Kollak \& Schmidt, 2015). Unter diese Kategorien fallen bei der GLESA-Analyse z.B. das Sehen von Bedürfnissen, Empathie und Respekt als Erwartungen, die von den Befragten bezogen auf die Pflege von LSBT*I-Personen ausgeführt wurden und keine explizite LSB-Spezifik aufweisen. Als LSB-spezifische Kategorien - entwickelt aus der Sachdimension „,(Gute) Pflege von LSBT*ISenior*innen“ - konnten zehn Kernkategorien mit weiteren elf untergeordneten Kategorien differenziert werden, die jeweils kursiv markiert wurden. Direkte Zitate werden in Anführungszeichen aufgeführt.

\section{Verständnis unterschiedlicher Lebenswelten}

Den Auskünften der Befragten zufolge bewirkt erst ein tieferes Verständnis der Lebenswelten von schwulen und lesbischen Senior*innen, dass diese und deren Angehörige sich nicht verstecken müssen und gute Lebensbedingungen auch in Pflegeeinrichtungen vorfinden. Kenntnisse zu den Lebenswelten von älteren LSBT-Personen sind für die Bewohner*innen des LoV unerlässlich für eine „gute Pflege“ von LSBT-Personen. Für die meisten Befragten - für einige Bewohner*innen und vor allem aus Sicht der Mitarbeitenden - war es daher entscheidend, dass ein Teil des Betreuungs- und Pflegepersonals selber schwul, lesbisch, bisexuell, trans* oder intergeschlechtlich ist. Nach Meinung der schwulen befragten Mitarbeitenden sollte mindestens ein/eine 
Hauptamtliche/r im Team selbst schwul oder lesbisch sein und Einfluss auf die Organisation oder die Praxen haben:

»Eben weil es sehr viel mit Vertrauen zu tun hat und weil man sich eben auskennen muss in der schwulen Lebenswelt im weitesten Sinne, ist das was Zentrales« (Hauptamtlicher, 52 J.: 120).

»Ich finde, es macht das leichter. (...) Und weil ich selber schwul bin, ist es so, wir brauchen da nichts zu erklären« (Hauptamtlicher, 54 J.: 329ff.).

Eine heterosexuelle Expertin verknüpft diese Forderungen nach LSBT*I-Personal zudem mit einer Kritik an Pflegeeinrichtungen, die im Hinblick auf sexuelle Orientierungen mehrheitlich nicht-integrativ seien (Hauptamtliche, 51 J.: 604).

Wenn ein schwul-lesbisches Klientel erreicht werden soll, raten insbesondere die Mitarbeitenden zu vertieften Kenntnissen der schwul-lesbischen Lebenswelt. Diese Lebenswelt sollte zudem vorrangig durch lesbische und schwule Menschen nähergebracht werden. Diesbezüglich verweisen die Hauptamtlichen auf ihre eigene sexuelle Orientierung und ihre beruflichen Erfahrungen. Sie empfinden einen „Vertrauensvorschuss“ bei den Bewohner*innen aufgrund der Zugehörigkeit zur schwul-lesbischen Community. Einige Expert*innen empfehlen Pflegediensten als günstige Voraussetzung für die Betreuung und Pflege von LSBT*I-Personen, dass die Leitung von schwulen Pflege-WG-Bewohnern selber schwul oder lesbisch sein sollte (Hauptamtlicher, $55 \mathrm{~J}$. : 176; Hauptamtliche, 59 J.: 290ff. und 307ff.). Je nach Gestalt der Pflegeeinrichtung deuteten diese Befragten jedoch an, dass flexible Lösungen ebenso möglich wären:

»Es war auch nie die Rede davon, dass sie 100 Prozent schwul sein sollte. Sondern größtenteils. Und das haben wir eingehalten. Und das finden wir selber auch wichtig, ne? (Hauptamtliche, 59 J.: 308).

LSBT*I-Beschäftigte sind nach Aussagen der Befragten als wichtige Ressourcen für Pflegeeinrichtungen $\mathrm{zu}$ begreifen. In diesem Zusammenhang sprachen sich die Befragten für die Unterstützung vergleichbarer Projekte aus (Hauptamtliche, 51 J.: 604).

\section{Pflege im „schwulen“ Mehrgenerationenhaus als Alternative}

Für die Bewohner*innen ist ein klassisches Pflegeheim keine Alternative im hohen Alter bzw. im Falle von Pflegebedürftigkeit. Diese Aussage wird zum einen für die Begründung des Einzugs in den LoV genannt und zum anderen als Konsequenz gezogen aus dem subjektiven Erleben von Versorgungseinrichtungen (vor allem der stationären Altenhilfe). Eine Bewohnerin zieht einen direkten Vergleich:

»Da [in einem normalen Pflegeheim] wird man einfach dadurch, dass man Minderheit ist, wieder zu so einer Exotenfigur. (...) Ja, das ist eben einfach so, dass über die ganzen Sachen hier kein Wort verloren wird « (Bew.in, 65 J.: 273). ${ }^{6}$

So sind die klassischen Alternativen aus Sicht der Befragten (zu) kostenintensiv und nehmen keine Rücksicht auf die Bedürfnisse von schwulen und lesbischen Bewohner*innen (Bew., 68 J.: 211ff.; Bew., 70 J.: 124ff.; Bew.in 65 J.: 273). In den Interviews werden Forderungen nach einem Fokus auf Minderheiten gestellt. Diese Forderungen verweisen auf Parallelen zu einer kultursensiblen Altenpflege. Respekt für ein „,von der Norm abweichende[s] Verhalten" und Berücksichtigung persönlicher Lebensgeschichte in Pflegeeinrichtungen werden von Bewohner*innen eingefordert (Bew., $33 \mathrm{~J}$. : 257; Bew., 49 J.: 529).

Die Expert*innen verweisen auf die Kooperation mit einem LSB-sensiblen Pflegedienst als Kriterium der guten Arbeit und des Erfolgs (Hauptamtlicher, 55 J., Hauptamtliche, 59 J.: 120ff.; Hauptamtlicher, 43 J.: 247ff.). Die enge Kooperationsarbeit zwischen Pflegedienst und Schwulenberatung im LoV drückt sich in einer gemeinsamen Akquise und in gemeinsamen Fallbesprechungen aus (Hauptamtliche, 59 J.: 164). Dabei verweisen die Interviewten auf die besonders enge Kooperation, die im Pflegealltag oft durch rechtliche Regelungen erschwert wird (Trennung von Vermieterund Pflegeanbieterleistungen etc.). Trotzdem ist eine Zusammenarbeit erlaubt, die eine von den Expert*innen als gut beurteilte Betreuung der Bewohner*innen gewährleistet, deren Lebensgeschichten so (besser) berücksichtigt werden können.

Einige Kategorien werden uneinheitlich aus den Daten beider Befragtengruppen entwickelt. So betonen vor allem die befragten Mitarbeitenden die Verknüpfung des Wohnprojekts mit einem spezifischen Angebot der Pflege in Form der "Pflege-WG“. Sie wird aber auch von den Bewohner*innen als sinnvolle Ergänzung des LoV wahrgenommen, die eine stationäre Versorgung vermeidet (Bew., 68 J.: 588; Hauptamtlicher, 54 J.: 515) und mögliche pflegerische Dienste bis zum Tod (Hauptamtliche, 59 J.: 602) übernimmt. In diesem Zusammenhang wird von einigen Befragten die Bedeutung des pflegerischen Settings inklusive der sozialen Netzwerke in der WG hervorgehoben, die eine präventive Wirkung ermöglicht (Hauptamtliche, 59 J.: 608; Bew.in 65 J.: 51). Einige der Interviewten betonen 
in diesem Zusammenhang eine Überlegenheit von PflegeWGs gegenüber üblichen stationären Pflegeeinrichtungen aufgrund eines höheren Individualitätsbezuges und der Ähnlichkeit zu familiären Lebensverhältnissen (Bew.Pflege-WG, 68 J.: 211; Hauptamtliche, 59 J.: 534; 43, Hauptamtliche, 51 J.: 606). Auch der Umgang mit Fragen von Nähe und Distanz, die bei den schwulen Pflegebedürftigen mit Erfahrungen zum Umgang mit Sexualität und Beziehungen verknüpft werden, scheint den hauptamtlich Tätigen in Wohngemeinschaften einfacher zu sein (Hauptamtlicher, 55 J.: 24ff.; 251; Bew., 68 J.: 294).

Die gemeinsamen Interessen von Schwulenberatung, LoV, Pflege-WG und Pflegedienst ziehen auch „flexible Lösungen“ der Zusammenarbeit nach sich. So wird von den Interviewten die Vermittlerposition eines Angestellten, der bei der Schwulenberatung beschäftigt ist, als Besonderheit bzw. als Vorteil interprofessioneller Zusammenarbeit verstanden und begrüßt (Hauptamtliche, 59 J.: 164-166, 318; Hauptamtliche, 51 J.: 320; Bew.Pflege-WG, 68 J.: 97).

\section{Same, Same but Different}

Der „Lebensort Vielfalt“ wird dann als Leuchtturm für andere Projekte gehandelt, wenn das Projekt in die Versorgungslandschaft eingeordnet wird. Von einem befragten Bewohner ist er in diesem Zusammenhang ein Beispiel für andere Projekte, umLSBT*I-Pflegebedürftige in die Regelversorgung zu integrieren:

»Also früher hieß es immer, man kümmert sich nicht um die Privatsphäre der Menschen. Also Sexualität ist ihre Privatsache. Da hat sich keine Pflegeeinrichtung darum zu kümmern, ne? Aber inzwischen ist es doch so, (...) dass man sich vorstellen kann, dass Schwule in solchen Einrichtungen oder Lesben isoliert ... doch ein isoliertes Leben führen. Also ein bisschen isoliert von den andern. Und das will man aufbrechen. Und das geht nur über viele Jahre. (...) Also dazu ist auch das Haus Lebensort Vielfalt irgendwie dazu da, diese Idee in der Gesellschaft zu fördern, dass das auch auf andere Bereiche übergreift, weil wir immer wieder mit der Frage konfrontiert werden, wieso braucht ihr überhaupt so ein Haus, ne? Warum genügen euch nicht normale klassische Pflegeeinrichtungen? (...) Aber im Grunde geht es nur darum, mit dem Haus auch so Anstöße zu geben - Impulse, dass das auch ausstrahlt auf andere Bereiche« (Bew., 68 J.: 248).

Der Bewohner empfindet den Versuch, Fragen der Sexualität im Alter ins Private zu verlagern, als Mittel der Sanktionierung durch die Gesellschaft, die eine fehlende Sichtbarkeit gelebter Sexualität in Pflegeeinrichtungen nach sich zieht. Der LoV wird als Gegenbeispiel gesehen, das zu einer Enttabuisierung von Sexualität im Alter und zu (mehr) Sichtbarkeit beitragen soll nicht nur von schwul-lesbischen Menschen, sondern in Pflegeeinrichtungen generell. Eine Idealvorstellung ist für einige der Befragten eine Versorgungslandschaft, bei der ein Ort wie der LoV nicht mehr nötig sei (Bew., 68 J.: 250). Ein Mitarbeitender sieht das ähnlich (Hauptamtlicher, 43 J.: 471ff.) und verweist dabei auf die Vorteile einer gelebten Vielfalt auch für Nicht-Homosexuelle aufgrund der Bereicherung des Lebens und der Erweiterung von Horizonten (Hauptamtlicher, 43 J.: 197ff.).

Der Wunsch nach einer verstärkten Thematisierung von Sexualität gilt nicht allein für die LSBT*I-Community. Die Bewohner*innen sprechen von Gemeinsamkeiten durch die soziale Lebenslage und durch die Zugehörigkeit zur Gruppe der Älteren an (Bew.in, 65 J.: 121; Bew.in, 68 J.: 162). Sie benennen das negative Altersbild, die enge Konnotation von Alter und Verlust. Sie sprechen in diesem Zusammenhang von gemeinsamen Interessen, die auch die sexuelle Identität weniger wichtig erscheinen lassen (Bew.in, 66 J.: 260; Bew.in, 65 J.: 61).

Sowohl die Bewohner*innen als auch die Expert*innen betonen die soziale Lebenslage als bedeutsam für das gemeinsame Erleben des Alterns und die Erkenntnis von Gemeinsamkeiten sowie die Notwendigkeit sozialer Netzwerke, die von dem/der Einzelnen zu gestalten seien. So verweisen die Interviewten explizit darauf, dass eine Anbindung an eine (biologische) Familie keine Garantie für sozialen Kontakt im Alter nach sich zieht (Bew., 49 J.: 529). Ein Hauptamtlicher weist dabei auf Besonderheiten bei der sozialen Einbindung schwuler älterer Männer hin:

»Ich meine, in Deutschland ist es ja immer noch so bekanntermaßen, dass die meisten Menschen, die Pflegebedarf haben, von ihren ... von den Familien gepflegt werden. Und das ist nun mal grade eben bei schwulen Männern ja oft nicht mehr ... nicht möglich. Deswegen ist es da umso wichtiger, dass es ... dass die trotzdem auch eingebunden sind in so familienartigen Zusammenhängen, find ich. Also, dass man sich eingebunden fühlt in eine ... was auch immer für , ne Gemeinschaft« (Hauptamtlicher, 52 J.: 370).

\section{Thematisierungen von herausfordernden Situationen}

Vor allem Hauptamtliche (nur wenige Bewohner*innen) verweisen auf teilweise herausfordernde Situationen und Ereignisse in der „Pflege-WG“ mit schwulen Bewohnern, die hinsichtlich der Grenzen des Auslebens sexueller Fantasien und Wünsche Fragen aufwerfen. So berichten sie von direkten sexuellen Wünschen, die im Pflegeteam, 
aber auch in der Schwulenberatung Diskussionen nach sich ziehen. Einige Beispiele seien hierfür genannt, so geht es beispielsweise um sexuelle Übergriffe auf Mitarbeitende:

»Ich weiß, dass es auch in der Pflege zu Situationen kommt, wo sexuelle Anspielungen oder Anmachen, sage ich jetzt mal etwas platt, es dazu kommt, wo aber die Mitarbeiter, die davon betroffen sind, bisher sich zumindest abgrenzen konnten. (...) Und die eine Seite ist eben das, dass wir da von Seiten des Pflegedienstes da noch mal ,ne ganz klare Position beziehen zu dem Thema« (Hauptamtliche, 51 J.: 395ff.).

Ebenso wird thematisiert, inwiefern sexuelle Dienstleistungen in der „Pflege-WG“ zur Sprache gebracht und hierbei Grenzen gezogen bzw. diskutiert werden:

»Grundsätzlich haben sie die Möglichkeit, das Angebot [z.B. das Angebot des mobilen Besuchsdienstes, Anmerkung der Autor*innen]. ... hat dann mittlerweile schon den Dritten gehabt, aber bei ihm ist es halt eben so, der möchte dann immer so gerne zwischen die Beine fassen und ein bisschen mehr« (Hauptamtlicher, 55 J.: 331ff.).

»Also Escort, also es gab wohl schon auch Stricher da in der WG. Ich meine, es ist ihre eigene Wohnung. Jeder kann sich ... kann da Gäste sich einladen, wie er möchte. Es darf nur nicht dazu kommen natürlich, wie es bei jeder Wohngemeinschaft ist, dass die anderen Bewohner davon irgendwie gestört werden« (Hauptamtlicher, 52 J.: 390ff.).

Die Mitarbeitenden thematisieren solche Fragen in Fallbesprechungen des Teams, wenn es Unklarheiten im Umgang damit gibt oder persönliche Grenzen der Mitarbeiter*innen überschritten wurden. Dabei nutzen vor allem die Befragten, die selbst schwul oder lesbisch sind, eine direktere Sprache und auch die Terminologie der schwulen Bewohner. Auch wenn die befragten Expert*innen oft mit einem Schmunzeln über das sexuelle Verhalten der Bewohner erzählen, würden Übergriffe nicht ignoriert und Grenzen mit den Beteiligten im Team besprochen (Hauptamtlicher, 52 J.: 390ff.). Möglichkeiten zum Ausleben von Partnerschaften werden mitunter aktiv geschaffen (Bew., $70 \mathrm{~J}$ : 136). Weiterhin verdeutlichen die Ergebnisse der GLESA-Studie, dass ein offener Umgang mit Sexualität und Verbalisierung von sexuellem Begehren Platz in der Aus-, Fort- und Weiterbildung von Fachpersonen haben sollten; in der Praxis sollten kommunikative Trainings und Supervision dazu stattfinden (Hauptamtliche, 51 J.: 296ff. und 404).

\section{Grundlegende Erfordernisse}

Die Antworten einer guten Pflege, die keinen expliziten Bezug zur schwul-lesbischen Thematik aufweisen, geben einen Einblick in das Erleben von pflegerischen Regeldiensten. Denn es wird der Bedarf nach ausreichendem, qualifiziertem und kontinuierlichen Personal genannt. Die Befragten würden im Rahmen einer Befragung von spezifischen Wünschen von LSBT*I-Senior*innen nicht regelmäßig und mit hoher Relevanz nennen, wenn sie selbstverständlich wären und in der pflegerischen Praxis als erfüllt gälten. Vor diesem Hintergrund bewerteten die Befragten die „weitergehenden Erwartungen“ nach einer grundsätzlich sensiblen Pflege homosexueller Menschen als weitgehend unrealistisch aufgrund problematischer Rahmenbedingungen in der Pflege. Fragen zur Umsetzung milieuspezifischer Anforderungen in der Praxis greifen wir in der nachfolgenden Diskussion auf.

\section{DISKUSSION}

Im Folgenden werden wir die eingangs gestellten Fragen nach den Besonderheiten bei der Pflege von lesbischen und schwulen Senior*innen mit daraus abgeleiteten diversitätssensiblen Handlungsempfehlungen vorstellen. Dabei werden auf Gemeinsamkeiten und Differenzen zum Konzept der kultursensiblen Altenpflege hingewiesen.

In Hinsicht auf das Konzept der kultursensiblen Altenpflege können zentrale Kriterien einer guten Pflege von LSB als vergleichbar aufgefasst werden, wenn z.B. Kenntnisse bzw. das Wissen über „die Kultur“" in Bezug auf Schwule und Lesben als das Wissen über die Lebenswelt von LSB verstanden wird. Darunter sind die Kenntnisse über die LSB-Community mit ihren Infrastrukturen und Angeboten, das Wissen über Diskriminierungserfahrungen in der Vergangenheit sowie aktuell und im Pflegealltag sowie die Bedeutung von Sexualität (in diesem Fall bei schwulen Männern) zu verstehen. Unsere Erkenntnisse zu den von den Befragten beschriebenen Lebenslagen von Homosexuellen und ihren Lebensgeschichten decken sich weitgehend mit den im Konzept der kultursensiblen Altenpflege (Paillon, 2010) ausgeführten Bedeutung des „Gefühls von Heimat“. Die bereits von Lottmann \& Castro Varela (2016) dargestellten Besonderheiten des LoV verdeutlichen die von den Bewohner*innen genannte Erwartung, im LoV ,,so sein zu dürfen, wie man ist“", wo die sexuelle Orientierung ,kein Thema“ sei (ebd., p. 17). Jedoch ist der Heimatbegriff der kultursensiblen Altenpflege (nicht nur) für LSBT*IPflegebedürftige problematisch; so kann hier eher von Zugehörigkeit und Verbundenheit zu (anderen) LSBT*IPersonen - unter anderem aufgrund von ähnlich erlebten Diskriminierungserfahrungen - ausgegangen werden. 
Auch das soziale Netzwerk, das in der kultursensiblen Altenpflege besondere Relevanz erfährt, scheint aus Sicht der Befragten für die Situation von schwulen (und lesbischen) Pflegebedürftigen äußerst bedeutsam zu sein. Der in der Regel häufig belastete bzw. fehlende Kontakt zur Herkunftsfamilie (Lautmann, 2016, p. 30) erfordert die Berücksichtigung (u.a. bei der Angehörigenarbeit) des sozialen Umfelds in außerordentlichem Maße hier sind - auch in Abgrenzung zur kultursensiblen Pflege - Besonderheiten bei der Berücksichtigung und Relevanz von wahlfamilialen Beziehungen (bspw. die lesbischen Freundinnen und Communities einer lesbischen Pflegebedürftigen) $\mathrm{zu}$ differenzieren. $\mathrm{Zu}$ der Angehörigenarbeit mit pflegebedürftigen LSBT*ISenior*innen besteht noch erheblicher Forschungsbedarf, der von der GLESA-Studie nicht adressiert wurde.

In Bezug auf die Sprache ist bei der Pflege von Schwulen und Lesben der milieuspezifische Humor oder entsprechende Begrifflichkeiten (z.B. schwul/lesbisch oder homosexuell) relevant. Hier kommt der im Konzept der Kultursensibilität genannten „Haltung gegenüber Besonderheiten der Kultur" eine entscheidende Bedeutung zu, die eine Offenheit des Pflegepersonals gegenüber pflegebedürftigen Schwulen und Lesben und einen kontextbezogenen Gebrauch von Begrifflichkeiten erforderlich macht. Bei theoretischen Übereinstimmungen des Konzepts der kultursensiblen Altenpflege für LSBPflegebedürftige ist hier von Defiziten bei der Anwendung und Übersetzung in der Praxis auszugehen.

Auch die in den Ergebnissen dargelegten Berichte der Befragten über Tabus in der pflegerischen Versorgung in Bezug auf LSB und fehlenden Sichtbarkeiten von LSB-Pflegebedürftigen in der Regelversorgung erfordern eine außerordentliche Kompetenz zur Differenzierung, wenn an dem Begriff der kultursensiblen Altenpflege festgehalten werden soll. Angesichts der Rahmenbedingungen in der Pflege zeigen sich doch erhebliche Zweifel, dass eine individualitätsorientierte Pflege diesen Anforderungen nachkommen kann. Zumal deutschen Pflegeheimen bereits unabhängig von der sexuellen oder geschlechtlichen Identität grundsätzliche Probleme bei der Berücksichtigung von Biografie und Lebensgeschichte beschieden werden und eine individuelle Ressourcen- und Resilienzförderung in pflegerischen stationären Einrichtungen ,,auf der Strecke bleibt" (Frieling-Sonnenberg, 2016, p. 70). Deshalb überrascht es beispielsweise nicht, dass in einer Berliner Umfrage die Hälfte der befragten Pflegeeinrichtungen es selbst als notwendig erachtet, LSB-Themen stärker zu behandeln (vgl. Schröder et al., 2012). Die von den GLESA-Befragten stets eingeforderte Maßgabe, in Pflegeeinrichtungen ein entsprechend sensibilisiertes Personal vorzufinden, wird oftmals gleichlautend damit verstanden, dass vor allem LSB-Personal selbst eine gewisse Sensibilität mitbringt (das wird angesichts der heterogenen Lebenswelten von LSBT*I Personen jedoch nicht regelmäßig der Fall sein). Vor diesem Hintergrund ist LSB-Pflegepersonal in pflegerischen Einrichtungen unbedingt als wichtige Ressource zu betrachten - das gilt insbesondere auch im Zusammenspiel mit dem nicht-pflegerischen Personal wie Sozialarbeiter*innen oder Verwaltungsangestellten. LSB-Personal kann oftmals die meist vorhandenen Lebensweltkenntnisse von älteren LSBT*I-Personen für eine „gute Pflege“ von LSBT*I einbringen, den Teilhabebegriff in der Pflege mit Leben füllen und als Ansprechpartner*innen tätig sein - sehr ähnlich zur kultursensiblen Altenpflege, bei der Fachkräfte mit transkulturellen Erfahrungen als „Mittler“ zwischen Kolleg*innen und Patient*innen fungieren können (Rommelsbacher, 2016, p. 216). Das Einsetzen der „Ressource LSB-Personal“ im eigenen Unternehmen ist aus unserer Sicht daher als konkrete Handlungsempfehlung zu verstehen.

Doch auch bei der Thematik der sexuellen Vielfalt in der Pflege gilt es wie bei der kultursensiblen Altenpflege zu beachten, dass diese spezifische personelle Ressource nicht (wie z.B. über eine*n LSBT*I-Beauftragte*n) dafür instrumentalisiert wird, dass der Betrieb als solches sich dem Thema „nicht stellen“ muss, es als „erledigt“ erklärt oder Heterosexuelle aus der Verantwortung entlassen werden (ebd., p. 216f.). Aus diesem Grund und weil Arbeitgeber*innen aufgrund des Allgemeinen Gleichstellungsgesetzes in der Personalauswahl weder positivnochnegativhinsichtlichdersexuellenOrientierung diskriminieren dürfen bzw. sollen, ist ferner zu empfehlen, über Schulungs- und Fortbildungsmaßnahmen das gesamte Personal für die Lebenswelten und Bedarfe der $\mathrm{LSB}(\mathrm{T} * \mathrm{I})$-Pflegebedürftigen zu sensibilisieren.

In Anbetracht der Vielfalt von Diversitätsdimensionen in der Lebenswelt LSBT*Ierscheint eine Weiterentwicklung derkultursensiblen Altenpflegezueinermilieuspezifischen und diversitätssensiblen Pflege notwendig, wenn diese Maßgaben der Intersektionalität zu berücksichtigen in der Lage wäre (vgl. Walgenbach, 2012: 11; Crenshaw, 1989). Es sei an dieser Stelle darauf verwiesen, dass auch schon für die kultursensible Altenpflege eine Ergänzung um „lebensweltliche und biografische Aspekte“ für eine „diversitätssensible Pflege“ als wünschenswert betrachtet werden (Tezcan-Güntekin \& Razum, 2017, p. 4). Mit einem stärker intersektional verorteten diversitätssensiblen Konzept in der Pflege könnten zudem „zu eng“ verstandene oder konzipierte Angebote für die jeweiligen Bevölkerungsgruppen (Stichwort: LoV als „Schwulen-Ghetto“?) integrativer diskutiert werden (ebd., p. 7). Gleichwohl darf dabei die gezielte Berücksichtigung obiger Diskriminierungspotenziale z.B. in stationären Pflegeeinrichtungen darüber nicht untergraben werden, denn die „Begegnung mit dem 
Anderen im Anderen" muss ein zentraler Aspekt bei einer diversitätssensiblen Altenpflege bleiben (Gerlach \& Schupp, 2016, p. 27f.).

In dieser Hinsicht kann das Berliner Projekt LoV grundsätzlich durchaus als Vorbild für weitere Wohnund Pflegeprojekte und Pflegeeinrichtungen verstanden werden. Doch werden einzelne Wohn- und Pflegeprojekte kaum eine zufriedenstellende und pflegerische Versorgung in eher ländlichen Räumen gewährleisten können. Die Autor*innen halten daher internationale und innovative Beispiele aus der Praxis wie den „Pink Passkey®“ für vielversprechend. Hierbei handelt es sich um ein niederländisches Zertifikat und Auditverfahren, welches in Regeldiensten pflegerischer Versorgung sicherstellen will, dass lesbische Frauen, schwule Männer, Bisexuelle und Trans*Personen (LSBT*) bei Pflegebedürftigkeit eine gute Versorgung erhalten. Es ist bislang nur in zwei deutschen Pflegeheimen implementiert worden, weshalb die Autor*innen empfehlen, ein Zertifikat für den deutschen Markt systematisch einzuführen - jedoch mit einer expliziten Berücksichtigung der Heterogenität und Diversität von LSBT*I-Personen (vgl. Linschoten, Lottmann, Lauscher, 2016).

Nun könnte eingeworfen werden, dass durch die zunehmende Gleichstellung von Schwulen und Lesben eine spezifische Sensibilisierung des Pflegepersonals für die Situation von LSBT*I irrelevant werde. Die o.g. Ausgangssituation bestehender Diskriminierungen im Alltag bleibt aber auch für spätere Generationen von LSBT*I-Senior*innen weitgehend bestehen-insbesondere für trans* und intergeschlechtliche Senior*innen, die mit einer ungleich stärkeren Unsichtbarkeit in der Gesellschaft kämpfen und deren Erfahrungen mit medizinischen Einrichtungen im Vergleich zu LSB-Pflegebedürftigen über den Lebensverlauf häufig ungleich negativer ausfallen.

Aufgrund der o.g. Konzentration des Forschungsdesigns auf allgemeine pflegerische Fragestellungen konnten im Rahmen dieser Studie nur erste spezifische Bedarfe dieser Bevölkerungsgruppe aufgezeigt und mit Beispielen illustriert werden. Diese machen die Notwendigkeit einer diversitätssensiblen Pflege deutlich. Um einen intersektionalen Blick und eine Untersuchung von Überkreuzungen von Diskriminierungsmerkmalen zu gewährleisten, der eine stärkere Differenzierung von heterogenen LSBT*I-Lebenswelten erlauben würde, sind weitere Untersuchungen notwendig insbesondere hinsichtlich der Situation von transund intergeschlechtlichen Pflegebedürftigen. Auch im Hinblick auf die biografischen Perspektiven der Betroffenen sind weitere Studien erforderlich. Diese wird die Forscher*innengruppe im Rahmen des Nachfolgeprojekts „Gleichgeschlechtliche Lebensweisen und Pflege im Alter" (GLEPA) $)^{7}$ fokussieren.

\section{FUSSNOTEN}

1. Da in der Studie vor allem schwule und lesbische Personen befragt wurden, konzentriert sich der Artikel auf Aussagen zu den Bedürfnissen dieser Gruppe.

2. GLESA ist ein vom Institut für angewandte Forschung Berlin (IFAF) finanziertes Kooperationsprojekt der Alice Salomon Hochschule Berlin und der Hochschule für Wirtschaft und Recht Berlin (Laufzeit: 1.04.2013 bis 31.05.2015).

3. Die Schreibweise mit einem * bietet zu den bekannten Geschlechtsidentitäten eine weitere, offene Kategorie für Menschen, die sich einer Geschlechterdichotomie entziehen möchten.

4. Als Cisgender werden Menschen bezeichnet, deren Geschlechtsidentität demjenigen Geschlecht entspricht, das ihnen bei der Geburt zugewiesen wurde.

5. Bei den Äußerungen der Expert*innen können aufgrund beruflicher oder ideeller „Zugehörigkeit zum Wohnprojekt" und dem Wunsch einer möglichst positiven Darstellung nach außen Aspekte der Befangenheit erkennbar werden, auf die hier hingewiesen wird. Dem wird durch eine Kategorisierung nach Mayring, die niemals auf nur einem oder wenigen Codes beruht, entgegengewirkt.

6. Anmerkung zur Transkription: Zwecks besserer Lesbarkeit werden Betonungen der Befragten (durch Unterstreichen) und Auslassungen (durch Klammern) angezeigt; Dialekte wurden erhalten, Namen oder Orte anonymisiert.

7. https://www.ash-berlin.eu/forschung/ forschungsprojekte-a-z/glepa/ (09/2017)

\section{Literatur}

Adelman, M. (2010). Groundbreaking study on LGBT aging: Need for housing, services and support. Abgerufen am 21.01.2018 http://openhouse-sf.org/wp-content/uploads/2010/01/RevisedGroundbreaking-Study-on-LGBT-Aging.pdf

Bischoff-Wanner, C. (2002). Empathie in der Pflege: Begriffserklärung und Entwicklung eines Rahmenmodells. Bern: Huber.

Bochow, M. (2008). Schwule Männer im dritten Lebensalter. Wohnmodelle und Forderungen. Respekt, 16.
Bochow, M. (2005). Ich bin doch schwul und will das immer bleiben. Hamburg: Männerschwarm.

Brennan-Ing, M., Liz, S., Larson, B., \& Karpiak, S. (2014). Social Care Net-works and Older LGBT Adults: Challenges for the Future. Journal of Homosexuality, 61(1), 21-52. doi:10.1080/00918369.2 013.835235

Bundesministerium für Familie, Senioren, Frauen und Jugend (2016). Die Charta der Rechte hilfe- und pflegebedürftiger Menschen. 
Abgerufen am 21.01.2018 https://www.pflege-charta.de/de/ startseite.html

Crenshaw, K. (1989). Demarginalizing the Intersection of Race and Sex: A Black Feminist Critique of Antidiscrimination Doctrine. The University of Chicago Legal Forum, 139-167.

Dömling, G. (2012). Kennzeichen kultursensibler Pflege. Wissenschaftliche Forschungsarbeit. Diakonie Deutschland Evangelischer Bundesverband. Berlin. Abgerufen am 21.01.2018 www.fh-diakonie.de/obj/Bilder_und_Dokumente/DiakonieCare/ FH-D_DiakonieCare_Doemling-G_Kennzeichen-kultursensiblerPflege_lang.pdf

Feil, N. (1967). Group therapy in a home for the aged. The Gerontologist, 7(3), 192-195.

Feil, N., Sutton, E., \& Johnson, F. (2014). Trainingsprogramm Validation. München: Reinhardt.

Fredriksen-Goldsen, K., \& Muraco, A. (2010). Aging and sexual orientation: A 25-year review of the literature. Research on Aging, 32(3), 372-413.

Frieling-Sonnenberg, W. (2016). Bedeutung der Lebensgeschichte für Gesundheit und Krankheit im (pflegebedürftigen) Alter - Zur Frage einer krankheitsfördernden Dynamik in Pflegeheimen und deren Anachronismus. In: R. Lottmann, R. Lautmann, \& M. d. M. Castro Varela (Hrsg.), Homosexualität_en und Alter(n) (pp. 69-80). Wiesbaden: Springer VS.

Gardner, A. T., de Vries, B., \& Mockus, D. S. (2013). Aging out in the desert: Disclosure acceptance, and service use among midlife and older lesbians and gay men. Journal of Homosexuality, 61(1), 129-144. doi:10.1080/00918369.2013.835240

Gerlach, H. (2002). Wie erleben homosexuelle Männer pflegerische Situationen? Pflegezeitschrift, 55(9/10), 2-6.

Gerlach, H. \& Schupp, M. (2016). Lebenslagen, Partizipation und gesundheitlich-/pflegerische Versorgung älterer Lesben und Schwuler in Deutschland: Expertise zum Siebten Altenbericht der Bundesregierung. Berlin: Deutsches Zentrum für Altersfragen.

Hoffmann-Riem, C. (1984). Das adoptierte Kind. Familienleben mit doppelter Elternschaft. München: Wilhelm Fink.

Jackson, N. C.; Johnson, M. J. \& Roberts, R. (2008). The Potential Impact of Discrimination Fears of Older Gays, Lesbians, Bisexuals and Transgender Individuals Living in Small- to Moderate-Sized Cities on Long-Term Health Care. Journal of Homosexuality, 54(3), 325-339. doi:10.1080/00918360801982298

Kitwood, T. (2000). Der personenzentrierte Ansatz im Umgang mit verwirrten Menschen. Bern: Huber.

Konkret Consult Ruhr (2008). Leitfaden zur Selbstbewertung auf Grundlage der Charta der Rechte hilfe- und pflegebedürftiger Menschen - Eine Handlungshilfe zur Umsetzung der Pflege-Charta in stationären Pflegeeinrichtungen. Abgerufen am 21.01.2018 https:// www.bmfsfj.de/blob/77440/177365c873d674ae168d4ebbd5f0525d/ leitfaden-selbstbewertung-charta-stationaere-einrichtungen-data.pdf

Kollak, I., \& Schmidt, S. (2015). Fallübungen Care und Case Management. Berlin: Springer.

Kollak, I., \& Küpper, H. (1997). Multikulturalität am Beispiel des Neuköllner Krankenhauses in Berlin. In: C. Uzarewicz, \& G. Piechotta (Hrsg.), Transkulturelle Pflege (pp. 115-131). Berlin: Verlag für Wissenschaft und Bildung.
Linschoten, M., Lottmann, R., \& Lauscher, F. (2016). »The Pink Passkey ${ }^{\circledR}$ «- ein Zertifikat für die Verbesserung der Akzeptanz von LSBT*I-Pflegebedürftigen in Pflegeeinrichtungen. In: R. Lottmann, R. Lautmann, \& M. d. M. Castro Varela (Hrsg.), Homosexualität_ en und Alter(n) (pp. 233-241). Wiesbaden: Springer VS.

Lautmann, R. (2016). Die soziokulturelle Lebensqualität von Lesben und Schwulen im Alter. In: R. Lottmann, R. Lautmann, \& M. d. M. Castro Varela (Hrsg.), Homosexualität_en und Alter(n) (pp. 15-50). Wiesbaden: Springer VS.

Lottmann, R. (2016). Mehr als ein Leuchtturm? Der „Lebensort Vielfalt" - ein Wohnprojekt für ältere Schwule, Lesben und Heterosexuelle. In: R. Lottmann, R. Lautmann, \& M. d. M. Castro Varela (Hrsg.), Homosexualität_en und Alter(n) (pp. 86-110). Wiesbaden: Springer VS.

Lottmann, R., \& Castro Varela, M. d. M. (2016). LSBT*I (k)ein Thema für die Altersforschung - Ausgewählte Befunde eines Forschungsprojekts. Informationsdienst Altersfragen, 43, 12-20.

Lottmann, R., \& Lautmann, R. (2015). Queer und Alter(n) - zum Forschungsstand. In: F. Schmidt, A. C. Schondelmayer, \& U. Schröder (Hrsg.), Selbstbestimmung und Anerkennung sexueller und geschlechtlicher Vielfalt. Lebenswirklichkeiten, Forschungsergebnisse und Bildungsbausteine (pp. 337-354). Wiesbaden: Springer VS.

LoV (2017). Lebensort Vielfalt (LoV). Abgerufen am 13.04.2017 https://www.schwulenberatungberlin.de/lebensort-vielfalt

Mayring, P. (2007). Qualitative Inhaltsanalyse. Grundlagen und Techniken. Weinheim: Beltz.

Meuser, M., \& Nagel, U. (2005). ExpertInneninterviews vielfach erprobt, wenig bedacht. Ein Beitrag zur qualitativen Methodendiskussion. In: A. Bogner, B. Littig, \& W. Menz (Hrsg.), Das Experteninterview. Theorie, Methode, Anwendung (pp. 7193). Opladen: Leske \& Budrich.

Meerendonk, B. van de, Adriansen, R., \& Vanwesenbeeck, I. (2003). Op weg naar een vrolijke herfst? Zorgbehoeften van en zorgverlening aan lesbische en homoseksuele ouderen in Nederland: onderzoek in het kader van het project 'vrolijke herfst'. Utrecht: Rutgers Nisso Groep.

Paillon, M. (2010). Kultursensible Altenpflege. Ideensammlung mit Fokus Demenz. München: Ernst Reinhardt.

Reimann, K., \& Lasch, V. (2006). Differenzierte Lebenslagen im Alter. Der Einfluss sexueller Orientierung am Beispiel homosexueller Männer. Zeitschrift für Gerontologie und Geriatrie, 39(1), 13-21. doi:10.1007/s00391-006-0364-8

Rommelsbacher, B. (2016). Pflege in einem multikulturellen Umfeld. In: B. Rommelsbacher, \& I. Kollak (Hrsg.), Interkulturelle Perspektiven für das Sozial- und Gesundheitswesen (pp. 197-216). Frankfurt/M.: Mabuse.

Tezcan-Güntekin, H., \& Razum, O. (2017). Pflege von Menschen mit Migrationshintergrund. In: K. Jacobs, A. Kuhlmey, S. Greß, A. Schwinger, \& J. Klauber (Eds), Pflege-Report 2017 - Schwerpunkt: Die Pflegebedürftigen und ihre Versorgung. Stuttgart: Schattauer.

Schröder U. B., Schondelmayer A. C., \& Scheffler, D. (2012). GesamtevaluationzurInitiative,,BerlintritteinfürSelbstbestimmung und Akzeptanz sexueller Vielfalt”. Ergebnisbericht. Landesstelle für Gleichbehandlung gegen Diskriminierung (Hrsg.), Dokumente 
lesbisch-schwuler Emanzipation Nr. 28. Abgerufen am 21.01.2018 https://www.bmfsfj.de/blob/77440/177365c873d674a e168d4ebbd5f0525d/leitfaden-selbstbewertung-charta-stationaereeinrichtungen-data.pdf

Schröder, U. B., \& Scheffler, D. (2015). „Bei uns gibt es dieses Problem nicht" - Leitlinien als Impuls für Veränderungen im gesellschaftlichen Umfeld. Wahrnehmung älterer LSBT-Menschen und die Wirkung der Berliner Seniorenleitlinien. In: F. Schmidt, A. C. Schondelmayer, \& U. Schröder (Hrsg.), Selbstbestimmung und Anerkennung sexueller und geschlechtlicher Vielfalt (pp. 319336). Wiesbaden: Springer VS.

Stummer, G. (2014). Kultursensible Pflege für Lesben und Schwule. Informationen für die Professionelle Altenpflege. Köln: Schriftenreihe des Rubicon e. V.: Kultursensible Pflege für Lesben und Schwule in NRW. Abgerufen am 21.01.2018 http:// www.rubicon-koeln.de/fileadmin/user_upload/Broschuere_ Kultursensible_Pflege_fuer_Lesben_und_Schwule.pdf

Unterforsthuber, A., \& Franz, H. (2004). Unterm Regenbogen Lesben und Schwule in München. Landeshauptstadt München Koordinierungsstelle für gleichgeschlechtliche Lebensweisen (Ed.). Abgerufen am 21.01.2018 ttps://www.muenchen.de/ rathaus/Stadtverwaltung/Direktorium/Koordinierungsstelle-fuergleichgeschlechtliche-Lebensweisen/Publikationen.html

Walgenbach, K. (2012): Intersektionalität - eine Einführung. Abgerufen am 1.10.2017 http://portal-intersektionalitaet.de/theoriebildung/ ueberblickstexte/walgenbach-einfuehrung/

White, J. T., \& Gendron, T. L. (2016). LGBT elders in nursing homes, long-term care facilities, and residential communities. In: D. A. Harley, \& P. B. Teaster (Hrsg.), Handbook of LGBT elders. An interdisciplinary approach to principles, practices, and policies (pp. 417-438). London: Springer.

Witzel, A. (2000). Das problemzentrierte Interview. Forum Qualitative Sozialforschung, 1(1). Abgerufen am 1.10.2017 http://www. qualitative-research.net/index.php/fqs/article/view/11 32/2520 